\title{
Esophageal Perforation Management: A Single-Center Experience
}

\author{
Ramzi Addas, Jean Berjaud, Claire Renaud, Pierre Berthoumieu, Marcel Dahan, \\ Laurent Brouchet
}

Department of Thoracic Surgery, Larrey Hospital, Toulouse, France.

Email: ramziaddas@yahoo.com,dahan.marcel@gmail.com, brouchet.l@chu-toulouse.fr

Received September $20^{\text {th }}, 2012$; revised October $29^{\text {th }}, 2012$; accepted November $16^{\text {th }}, 2012$

\begin{abstract}
Background: Esophageal perforation is a rare and potentially life-threatening condition requiring urgent management. Successful therapy depends on the underlying etiology, clinical presentation, the time between rupture and diagnosis, the extent of the rupture and the underlying health of the patient. Method: From 2005 to 2012, the author retrospectively analyzed 36 patients treated for esophageal perforation. Data were evaluated for cause of perforation, symptoms, comorbidities, the method of diagnosis, delay in diagnosis, therapeutic regimen, complications, hospital stay, follow-up and mortality. Results: The cause of perforation were iatrogenic in 14 cases (38.8\%), foreign body ingestion in 11 (30.5\%), spontaneous in 9 (25\%), chest trauma in $1(2.8 \%)$ and esophageal cancer in 1 case $(2.8 \%)$. The most frequent signs and symptoms were chest pain in 27 cases (75\%), fever in 15 (41.6\%), dysphagia in 11 (30.5\%), mediastinitis in 9 (25\%) and vomiting in 8 (22\%). The treatment included surgery in 26 cases (72.2\%) which consists of thoracotomy (right or left), with or without esophageal suturing, washing, drainage with three chest tubes, jejunostomy and gastrostomy. The second group were patients treated medically in 10 cases (27.8\%), medical treatment includes nil per os (NPO), parenteral nutrition, intravenous antibiotics and observation. Complications include fever $(\mathrm{n}=14)$, auricular fibrillation $(n=7)$, esophageal fistula $(n=3)$, reoperation $(n=2)$, renal failure $(n=2)$, cerebrovascular accident $(n=1)$, pulmonary embolism $(n=1)$, pneumonia $(n=1)$ and deep vein thrombosis $(n=1)$. The average hospital stay for patients treated surgically was 36 days and for patients treated medically was 14.2 days. The overall mortality was $25 \%$ involving 8 patients treated surgically and 1 patient treated medically. Conclusion: The treatment method still must be chosen on an individual basis. Rapid diagnosis of this often life threatening condition is critical for expediting the choice of an optimal treatment strategy, whether surgical or non-surgical.
\end{abstract}

Keywords: Esophagus; Esophageal Perforation; Boerhaave’s Syndrome

\section{Introduction}

Esophageal perforation is an uncommon disease with high morbidity and mortality [1]. It has been regarded as the most serious injury of the digestive system. Several factors including the difficulty of accessing the esophagus, the lack of strong serosal layer, the unusual blood supply of the organ and the proximity of vital structures, all contribute to this conditions high morbidity and to a mortality rate of at least $20 \%[2,3]$. The spectrum of severity can vary from minimal leakage of air in the mediastinum to gross disruption and free drainage into the pleural cavity.

The importance of early diagnosis and prompt treatment is very important. The mortality rate rises up to $60 \%$ if there is delay in diagnosis and initiation of treatment. On the other hand this rate decreases to $10 \%$ if treatment started within 24 hours of perforation [4,5].
The scientific evidence that guides management of esophageal perforation is based on retrospective studies. Randomized studies are non-existent. Treatment maybe medical (conservative) or surgical, depending on the cause, site, extent, symptoms and radiological findings [6].

The management of esophageal perforations remains challenging and each case should be evaluated individually.

The aim of this study was to analyze the characteristics, treatment, and outcome of patients with esophageal perforation referred to our hospital during the last seven years.

\section{Patients and Methods}

A retrospective review was performed of patients with esophageal rupture treated at our institution between 2005 and 2012 (36 cases were included in the study). 
Esophageal fistula and Zinker diverticulum cases were excluded from the study. The following data were collected: patient age, sex, risk factors, delay in diagnosis, route of diagnosis, site of perforation, etiology of perforation, type of management, complications, hospital stay and outcome.

The program Excel was used for our statistical analysis. No other programs were used as the number of patients was small and a randomized study cannot be done.

All patients were assessed in our specialist hospital by an experienced thoracic surgeon. The status of the esophageal perforation was evaluated with an esophagogram and a computed tomography CT scan.

Patients who were unfit for definitive surgery or had minimal esophageal perforations without signs of sepsis, mediastinitis or pleural effusion were managed nonoperatively. Non-operative management includes 1) Nilby-mouth, 2) intravenous antibiotics, 3) high dependency/intensive care support, 4) gastric decompression (nasogastric tube) in some cases, and 5) nutritional support (parenteral).

Patients who were fit for surgery or had an esophageal rupture with sepsis, mediastinitis and pleural effusion underwent surgical intervention. Objectives of surgical management were 1) pleural and mediastinal decontamination, 2) debridement or resection of devitalized esophageal tissue, 3) primary repair of perforation (if possible), 4) drainage of pleural and mediastinal spaces, 5) gastric decompression, and 6) distal enteral feeding access. The operative approach was influenced by the patients general condition, suitability of the esophagus for primary repair and degree of intrathoracic contamination.

A thoracic approach was via thoracotomy (right or left), with or without esophageal suturing, washing, thoracic drainage with three chest tubes. In addition, patients underwent laparotomy for insertion of decompressing gastrostomy and feeding jejunostomy. VATS was not used as the condition is usually urgent and our surgeons preferred to do a thoracotomy for a better exposure.

The patients postoperative course includes intensive care unit (ICU) follow-up for at least 48 hours.

A daily observation of vital signs, clinical presentation, laboratory analysis (at least twice a week) and chest $\mathrm{X}$-rays.

A water soluble contrast esophagography is done one week after the intervention to confirm esophageal continuity before resuming a soft diet before discharge.

\section{Results}

During the last seven years there were 36 patients diagnosed as having esophageal rupture .There were 20 males (55.5\%) and 16 females (44.5\%) with mean age of 59.3 years(range from 17 to 91 years).
Most important comorbidities (Table 1) includes hypertension ( $(\mathrm{=}=9)$, tobacco smoking $(\mathrm{n}=7)$, hypercholesterolemia $(n=4)$ and alcoholism $(n=3)$.

The etiologies of the various perforations are illustrated in (Figure 1). Iatrogenic perforations constituted the most common cause including 14 patients (38\%). Iatrogenic causes were very variable including transesophageal echocardiography, esophagoscopy, mediastinoscopy, after chest tube insertion for pneumothorax, during gastric washing for Ehler-Danlos syndrome, esophageal dilatation, endoscopic cholangiopancreatography or during a surgical intervention. The second cause of esophageal perforation was foreign body ingestion in 11 cases (30.5\%), foreign bodies includes fish bones, chicken or pork bones and teeth. The third cause was spontaneous esophageal rupture (Boerhaave's syndrome) in 9 (25\%). Other causes include chest trauma by white weapon in 1 (2.7\%) and perforation due to esophageal tumor in 1 (2.7\%).

Patients diagnosed and treated within 24 hours of perforation were 23 patients (63.8\%).

At time of presentation, the great majority of patients (75\%) complained of chest pain. Other signs and symptoms (Figure 2) included fever (41.6\%), mediastinitis (25\%), dysphagia (30.5\%), and dyspnea (14\%).

Table 1. Patients comorbidities.

\begin{tabular}{cc}
\hline Comorbidity & Number of patients \\
\hline Hypertension & $9(25 \%)$ \\
Tobacco smoking & $7(19 \%)$ \\
Dyslipidemia & $4(11 \%)$ \\
Psychological problems & 4 \\
Ischemic cardiomyopathy & $3(8 \%)$ \\
Alcoholism & 3 \\
Chronic obstructive pulmonary disease & 3 \\
Myocardial infarction & $2(5.5 \%)$ \\
Auricular fibrillation & 2 \\
Hepatitis (B ,C) & 2 \\
Peripheral vascular disease & 2 \\
Diabetes Mellitus & 2 \\
Stroke & 2 \\
Asthma & $1(2 \%)$ \\
Heart valve replacement & 1 \\
Esophageal cancer & 1 \\
Esophagitis & 1 \\
Ehler-Danlos syndrome & \\
& 2 \\
\hline & 2 \\
\hline & \\
\hline
\end{tabular}




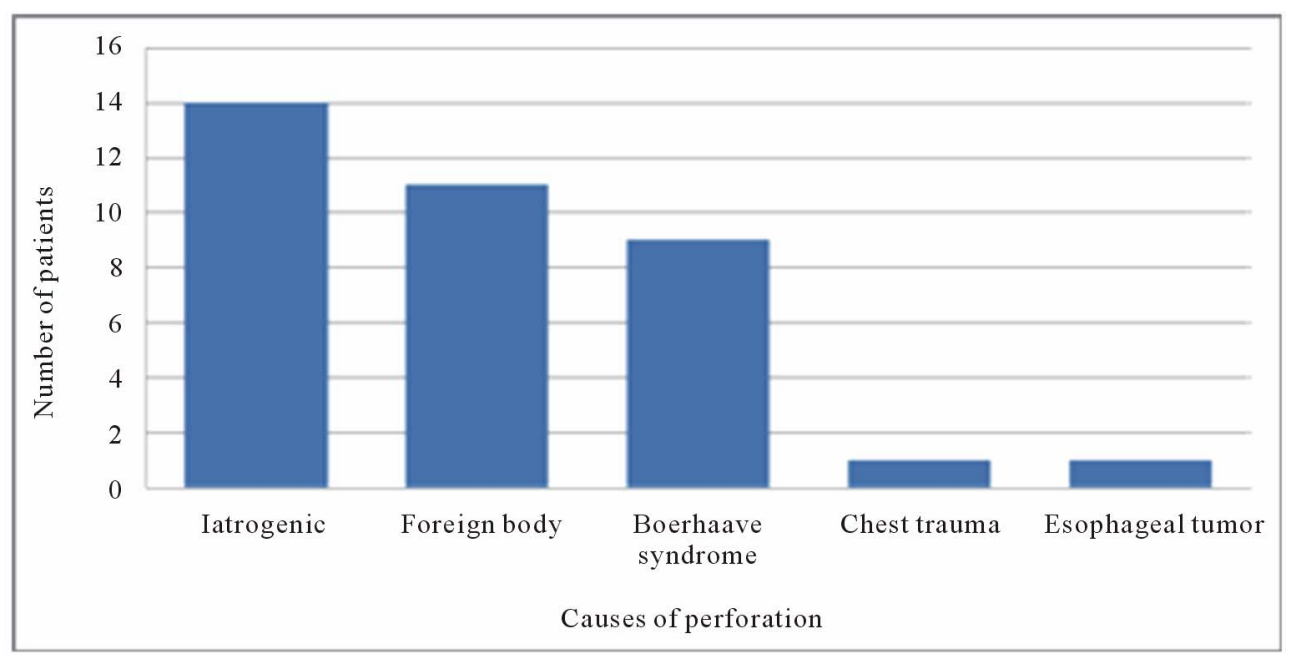

Figure 1. Etiology of esophageal perforations.

Plain chest X-rays were often suggestive of esophageal perforation, with subcutaneous emphysema, pneumothorax or pleural effusion. CT scan was realized in $78 \%$ of Patients $(n=28)$, esophagography in 24 patients $(66.6 \%)$ and esophageal fibroscopy in $11 \%$ of cases $(n=4)$.

\subsection{Medical Group}

In our study there were ten patients treated medically (27.7\%). Male to female ratio was $1: 1$ with a mean age of 54.6 years (from 17 to 91 years) (Table 2). Half of these patients had esophageal rupture due to foreign body ingestion. Three patients due to spontaneous rupture and two due to iatrogenic causes. Ninety percent of these patients presented with chest pain. CT scan was performed in all of them. In this group of patients 9/10 patients had the diagnosis of esophageal rupture within 24 hours of the perforation. There was one mortality which was a 91-year-old female which had esophageal rupture after a trans-esophageal echography. The perforation was complicated by mediastinitis and death after about one month of hospitalization. She was old and had many other risk factors (cerebrovascular accident, colon cancer, aortic valve replacement) and after discussion between the surgical team, anesthesiologists and her family we decided not to operate her. Otherwise the nine other patients that were treated medically were relatively young and in good general health. The perforation was diagnosed within 24 hours (except one of them).

The medical treatment was started immediately after the diagnosis. The evolution during hospitalization was satisfied with no mediastinitis or other major complications.

Reevaluation within one week of hospitalization with water soluble contrast esophagography was satisfied and patients were allowed to start alimentation per os starting by liquids and then increasing the consistency gradually.
Table 2. Comparison between surgically and medically treated patients (NB: percentages written beside the numbers are related to each group and not for the whole number of patients).

\begin{tabular}{|c|c|c|}
\hline Character & $\begin{array}{c}\text { Number of } \\
\text { patients } \\
\text { surgically treated }\end{array}$ & $\begin{array}{c}\text { Number of } \\
\text { patients } \\
\text { medically treated }\end{array}$ \\
\hline \multicolumn{3}{|l|}{ Sex } \\
\hline Male & 15 (57.7\%) & $5(50 \%)$ \\
\hline Female & $11(42.3 \%)$ & $5(50 \%)$ \\
\hline Age average & $\begin{array}{l}61 \text { years (range } \\
\text { from } 20 \text { to } 90 \text { ) }\end{array}$ & $\begin{array}{c}54.6 \text { years (from } 17 \\
\text { to } 91 \text { years) }\end{array}$ \\
\hline \multicolumn{3}{|l|}{ Signs and symptoms } \\
\hline Chest pain & $18(69 \%)$ & $9(90 \%)$ \\
\hline Fever & $14(54 \%)$ & $1(10 \%)$ \\
\hline Mediastinitis & $8(30.7 \%)$ & $1(10 \%)$ \\
\hline Dysphagia & 8 & $3(30 \%)$ \\
\hline Vomiting & 8 & - \\
\hline Dyspnea & $5(19 \%)$ & - \\
\hline Subcutaneous emphysema & $3(11.5 \%)$ & $1(10 \%)$ \\
\hline Nausea & 3 & - \\
\hline \multicolumn{3}{|l|}{ Causes } \\
\hline Iatrogenic & $12(46 \%)$ & $2(20 \%)$ \\
\hline Foreign body & $6(23 \%)$ & $5(50 \%)$ \\
\hline Spontaneous (Boerhaave’s) & 6 & $3(30 \%)$ \\
\hline Chest trauma & $1(3.8 \%)$ & - \\
\hline Esophageal cancer & 1 & - \\
\hline \multicolumn{3}{|l|}{ Complications } \\
\hline Fever & $12(46 \%)$ & $2(20 \%)$ \\
\hline Auricular fibrillation & $6(23 \%)$ & $1(10 \%)$ \\
\hline Esophageal fistula & $3(11.5 \%)$ & - \\
\hline Mediastinitis & $2(7.6 \%)$ & - \\
\hline Renal insufficiency & 2 & - \\
\hline Pneumonia & 2 & - \\
\hline Reoperation & $1(3.8 \%)$ & - \\
\hline Pulmonary embolism & 1 & - \\
\hline Deep vein thrombosis & 1 & - \\
\hline Urinary infection & 1 & - \\
\hline Hospital stay average & $\begin{array}{c}36 \text { days ( range } \\
\text { from } 10 \text { to } 79 \\
\text { days) }\end{array}$ & $\begin{array}{l}14.2 \text { days (range } \\
\text { from } 7 \text { to } 39 \text { days) }\end{array}$ \\
\hline Mortality & $8(30.7 \%)$ & $1(10 \%)$ \\
\hline
\end{tabular}




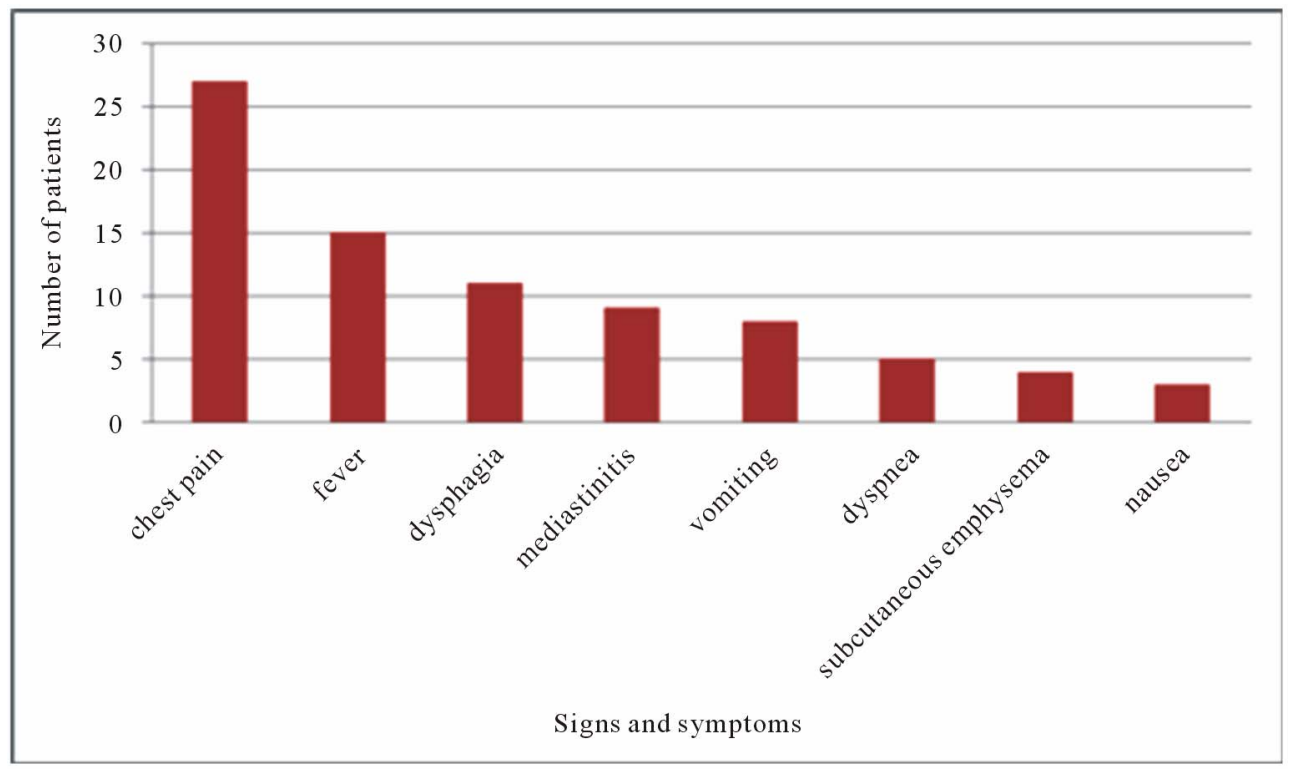

Figure 2. Signs and symptoms.

The average hospitalizations of this group of patients were 14.2 days (range from 7 to 39 days). They were all well at discharge and later on follow-up in clinic.

\subsection{Surgacal Group}

On the other hand, there were 26 patients treated surgically (72.3\%), there were 15 males (57.7\%) and 11 females (42.3\%) (Table 2). Age average was 61 years (from 20 to 90 years old).

Main risk factors for this group of patients were hypertension (26.9\%), myocardial infarction (7\%), Chronic obstructive pulmonary disease (11.5\%), tobacco smoking (23\%), dyslipidemia (11.5\%), esophageal cancer (7\%). The causes of esophageal perforations were iatrogenic (46.2\%), spontaneous (23.1\%), foreign body inhalation (23.1\%), chest trauma by a stab wound (3.8\%) and due to esophageal tumor (3.8\%).

Fourteen patients (53.8\%) were operated within 24 hours of the diagnosis. Most post-operative complications included fever (46\%), auricular fibrillation (23\%), pneumonia $(7.6 \%)$, re-intervention for surgical failure (7.6\%), renalinsufficiecy (7.6\%), pulmonaryembolism (3.8\%), deep vein thrombosis in 1 case.

Mortality was $30.7 \%$ (8 cases) in this group of patients, half of them had esophageal rupture due to iatrogenic reasons. The average hospital stay for that group of patients were 36 days (between 10 and 79 days).Long term follow-up for these patients was satisfied except for three patients that had mild dysphagia while eating solids.

If we look at the comparison between the two groups, we will find that most of the patients that had esophageal rupture due to iatrogenic causes were treated surgically. Another predisposing factor for surgical treatment was the presence of mediastinitis and pleural effusion. Complications were more common in patients treated surgically as well as the duration of hospitalization which was longer in patients treated surgically compared to patients treated medically.

\section{Discussion}

Perforation of the esophagus is a clinical entity with a recognized potential for morbidity and mortality. In a recent population based study in Iceland, the age standard incidence was 3.1/1,000,000/year [7]. Most patients are in their sixties, and esophageal perforation is slightly more common in males [3].

The etiology of esophageal perforation in the majority of patients (76\%) is either diagnostic or therapeutic instrumentation of the esophagus. Although management of esophageal rupture has advanced, it appears that the treatment of esophageal perforation will remain a challenge as the number of patients is increasing [8].

In our experience, the majority of perforations were iatrogenic in 14 cases (38.8\%).

The second cause is foreign body inhalation in 11 cases (30.5\%). Foreign bodies can cause esophageal perforation by direct penetration, pressure, chemical necrosis, or during endoscopic removal [9]. In the literature, they account for $7 \%$ to $14 \%$ of esophageal perforation [9]. The usual sites affected are the three natural anatomic narrowings: the cricopharyngeus, the crossing of the left main stem bronchus or aortic arch, and the gastroesophageal junction [10]. In a series of 2394 cases of retained esophageal foreign body reported from Hong Kong, perforation occurred in 25 cases (1\%) [10].

A wide variety of objects were retained in the esopha- 
gus but fish bones were the most common (60\%). Other foreign bodies are reported in the literature as well, for example coins, have perforated the esophagus, and fatal esophago-aortic perforation by a coin has been reported in a child of three old [11].

Clinical manifestation of foreign body perforation may be seen immediately or as late as 2 weeks later, as a gradual erosion of the impacted foreign body through the esophageal wall.

The third cause was Boerhaav's syndrome (spontaneous esophageal rupture) in 9 cases (25\%). The first description of spontaneous esophageal perforation was by the Dutch physician, Hermann Boerhaave in 1724. Boerhaavés syndrome (spontaneous rupture) results from an acute barotrauma with a sudden pressure rise in the esophagus at the occasion of protracted forceful vomiting in most cases (as in our study).

The other two cases were esophageal rupture due to knife stab and due to esophageal tumor.

For many years, spontaneous perforation was the primary etiology of esophageal perforations.

However, with the introduction of advanced endoscopic therapies, iatrogenic perforations have accounted for a larger proportion of thoracic esophageal injuries.

The diagnosis of esophageal rupture is challenging due to rarity of condition and non-specific signs and symptoms. The most consistent symptom of the esophageal injury is pain localized along the course of the esophagus [9]. However, up to one third of cases of perforated esophagus are atypical [9]. The commonest misdiagnosis are: myocardial infarction, pulmonary embolism, dissecting aneurysm and pancreatitis.

Diagnosis can be made using a chest X-ray (which can be normal in a lot of cases), CT scan (Figures 3 and 4) and Esophagogram (Figure 5). In our study $78 \%$ of the cases were diagnosed by CT scan $(\mathrm{n}=28)$. Minnich et al. has reported that esophagogram and CT scan are obtained in all patients to document whether the perforation is contained within the mediastinum as well as the presence or absence of a pleural or mediastinal fluid collection [12]. If there is extravasation of contrast into the pleural space, operation is recommended for drainage of the pleural space, decortication of the lung, debridement and drainage of the mediastinum, and possible esophageal repair with additional soft tissue coverage.

Treatment options include non-operative and operative procedures. Shenfine and Griffin had adopted algorithms for management of iatrogenic and spontaneous esophagus perforations [13].

No proves exists as to the best treatment, but there are four aims in management strategy: 1) Direct repair if possible, if not possible, functional or surgical isolation of the esophagus from the stomach; 2) Adequate drainage; 3) Appropriate antibiotics, 4) Adequate feeding [14].

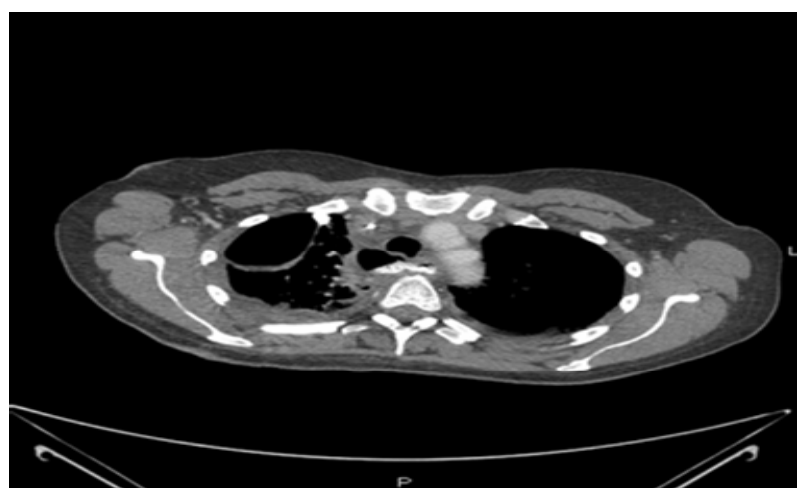

Figure 3. Esophageal perforation due to foreign body ingestion in a 54-year-old female, complicated with pleural effusion.

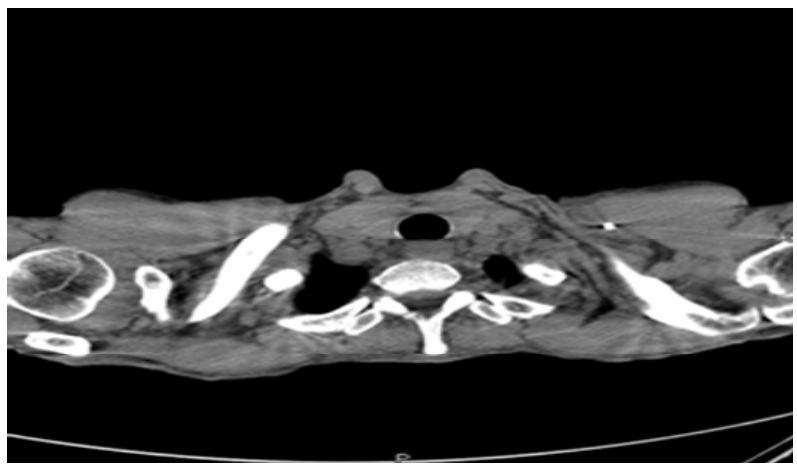

Figure 4. Esophageal perforation due to foreign body ingestion in a 51-year-old male, complicated with pneumomediastinum.

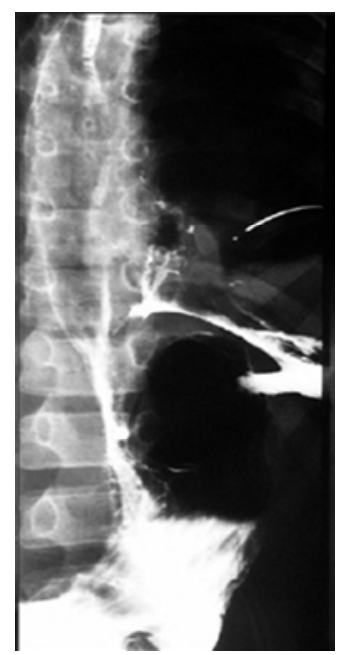

Figure 5. Esophagogram anteroposterior view showing extravasation of contrast from the distal third of esophagus.

Non-operative treatments are best with a contained perforation and the absence of clinical mediastinitis. It includes 1) Nil per os, 2) intravenous antibiotics, 3) nasogastric decompression, 4) enteral nutrition and 5) insertion of chest tube .Cameron et al. [15] proposed three 
criteria for nonoperative management: 1 ) perforation must be contained in the mediastinum and should be drained back into the esophagus; 2) there are mild symptoms; and 3) there should be minimal evidence of clinical sepsis. Wesdrop et al reported a series of 49 patients with endoscopic perforations treated non-operatively with a $6 \%$ mortality [16].

In our study, we found that patients that had esophageal perforation associated with mediastinitis and/or pleural effusion required surgical intervention.

The other type of treatment is the operative procedures. The choice of the operative strategy depends on the cause, location of injury, underlying esophageal diseases, time interval after perforation, extension of spillage, edge of wound, age of patient and the presence of comorbidities.

Many thoracic surgeons believe that primary repair is the gold standard for patients who present within the first 24 hours after perforation. Barrett et al reported in 1947 the suture repair of spontaneous esophageal rupture [17]. Since then, the role of surgical intervention in the management of esophageal injury has remained controversial. Bladergoen et al. reported that primary suture repair of esophageal perforations yields the most favorable results, particularly evident for perforations treated within 24 hours of symptoms (92\% survival) [4]. Survival was reduced when suturing occurred after 24 hours. The decision of drainage only versus drainage plus esophageal repair is determined intra-operatively by the quality of the esophageal tissue and the overall condition of the patient.

In fact, literature shows also that primary repair is possible regardless of time interval between perforation and surgery if esophageal tissue is repairable and wound edges are viable after necrosectomy and the size of defect is not greater than one third of the circumference of the esophagus. Jougon et al showed that a long interval before treatment does not preclude primary esophageal repair [18]. We should keep in mind, that the greater the delay in the diagnosis of perforation, the more edematous and necrotic is the esophageal wall. That will increase the difficulty of identifying the esophageal wall during dissection and direct repair can fail.

Other reported managements of esophageal rupture includes the usage of stents. Although not commonly used in our department the usage of esophageal stents for the treatment of esophageal perforation remain an additional option. Stents are used to treat some cases of esophageal perforation in the department of Gastroenterology in Toulouse, but they had lots of complications. For that reason we didn't use stents in the management of esophageal rupture in our department. Stents require close radiological and endoscopic follow-up to ensure no migration and continued coverage of the esophageal defect [19].
Different complications are reported regarding esophageal rupture. Gastroesophageal reflux disease is the main complaint of patients surviving esophageal rupture [20]. Although GERD is commonly considered as a sequel of the rupture and/or its treatment, GERD may also contribute to the origin of the rupture. This assumption has been already emphasized by Salo and colleagues, who concluded on the basis of a retrospective study of 5 patients that survivors of a spontaneous esophageal rupture, have a long-term severe disturbance of esophageal motility caused by chronic reflex esophagitis [21]. Another complication of esophageal rupture is motility disorders.

D'Journo et al. reported a motility disorders in 6 of 7 patients in their Study [22]. In the series of Salo et al., 4 of 5 patients were considered to have nonspecific esophageal motility disorder (NEMD).

In comparing the medical and surgical groups we find that medically treated patients were in a better general health, relatively younger and had less co-morbidities. Most of patients that had esophageal rupture due to iatrogenic causes were treated surgically. Another predisposing factor for surgical treatment was the presence of mediastinitis and pleural effusion. Of the 36 patients, nine had mediastinitis, only one of them was treated medically.

According to the delay in between the diagnosis and surgical intervention we didn't find a lot of difference in the outcome between patients operated within 24 hours of diagnosis and patients operated more than 24 hours after the diagnosis.

Limitations of this study include its retrospective nature. In addition, the incidence of esophageal perforation is low enough that it does not allow large sample sizes from a single institution.

\section{Conclusion}

The choice of treatment of esophageal perforation has to be done individually. Prompt diagnosis and appropriate treatment can allow ultimate survival for the patient with this challenging clinical problem.

\section{REFERENCES}

[1] K. K. Sng, A. J. Koh, N. C. Tan, S. M. Tan and K. H. Tay, "An Eastern Perspective on Oesophageal Perforation: A High Incidence of Ingested Bones,” ANZ Journal of Surgery, Vol. 78, No. 7, 2008, pp. 573-578. doi:10.1111/j.1445-2197.2008.04575.x

[2] P. Ryom, J. B. Ravn, L. Penninga, S. Schmidt, M. G. Iversen, P. Skov-Olsen and H. Kehlet, “Aetiology, Treatment and Mortality after Oesophageal Perforation in Denmark,” Danish Medical Bulletin, Vol. 58, No. 5, 2011, p. A4267. 
[3] P. Bhatia, D. Fortin, R. I. Inculet and R. A. Malthaner, "Current Concepts in the Management of Esophageal Perforations: A Twenty-Seven Year Canadian Experience,” The Annals of Thoracic Surgery, Vol. 92, No. 1, 2011, pp. 209-215. doi:10.1016/j.athoracsur.2011.03.131

[4] M. R. Bladergroen, J. E. Lowe and R. W. Postlethwait, "Diagnosis and Recommended Management of Esophageal Perforation and Rupture," The Annals of Thoracic Surgery, Vol. 42, No. 3, 1986, pp. 235-239. doi:10.1016/S0003-4975(10)62725-7

[5] M. G. Sarr, J. H. Pemberton and W. S. Payne, "Management of Instrumental Perforations of the Esophagus," Journal of Thoracic and Cardiovascular Surgery, Vol. 84, No. 2, 1982, pp. 211-218.

[6] K. Tsalis, K. Blouhos, D. Kapetanos, T. Kontakiotis and C. Lazaridis, "Conservative Management for an Esophageal Perforation in a Patient Presented with Delayed Diagnosis: A Case Report," Cases Journal, Vol. 2, 2009, p. 164. doi:10.1186/1757-1626-2-164

[7] H. Vidarsdottir, S. Blondal, H. Alfredsson, A. Geirsson and T. Gudbjartsson, "Oesophageal Perforations in Iceland: A Whole Population Study on Incidence, Aetiology and Surgical Outcome," The Thoracic and Cardiovascular Surgeon, Vol. 58, No. 8, 2010, pp. 476-480. doi:10.1055/s-0030-1250347

[8] V. Bresadola, G. Terrosu, A. Favero, et al., "Treatment of Perforation in the Healthy Esophagus: Analysis of 12 Cases," Langenbeck's Archives of Surgery, Vol. 393, No. 2, 2008, pp. 135-140. doi:10.1007/s00423-007-0234-X

[9] A. Kanowitz and V. Markovchick, “Oesophageal and Diaphragmatic Trauma,” In: P. Rosen, Ed., Emergency Medicine: Concepts and Clinical Practice, 4th Edition, Mosby, St. Louis, 1998, pp. 546-548.

[10] P. Nandi and G. B. Ong, "Foreign Body in the Oesophagus: Review of 2394 Cases,” British Journal of Surgery, Vol. 65, No. 1, 1978, pp. 5-9. doi:10.1002/bjs.1800650103

[11] M. Dahiya and J. S. Denton, "Esophagoaortic Perforation by Foreign Body (Coin) Causing Sudden Death in a 3Year-Old Child," American Journal of Forensic Medicine \& Pathology, Vol. 20, No. 2, 1999, pp. 184-188. doi:10.1097/00000433-199906000-00016

[12] D. J. Minnich, P. Yu, A. S. Bryant, D. Jarrar and R. J. Cerfolio, "Management of Thoracic Esophageal Perforations,” European Journal of Cardio-Thoracic Surgery, Vol. 40, No. 4, 2011, pp. 931-937.

[13] J. Schenfine and S. M. Griffin, "Oesophageal Emergencies,” In: S. M. Griffin and S. A. Raimes, Eds., Oeso- phagogastric Surgery-A Companion to Specialist Surgical Practice, 3rd Edition, Elsevier Saunders, Philadelphia, 2006, pp. 365-393.

[14] E. Teh, J. Edwards, J. Duffy and D. Beggs, “Boerhaave’s Syndrome: A Review of Management and Outcome,” Interactive CardioVasc Thoracic Surgery, Vol. 6, No. 5, 2007, pp. 640-643. doi:10.1510/icvts.2007.151936

[15] J. L. Cameron, R. F. Kieffer, T. R. Hendrix, D. G. Mehigan and R. R. Baker, "Selective Nonoperative Management of Contained Intrathoracic Esophageal Disruptions," The Annals of Thoracic Surgery, Vol. 27, No. 5, 1979, pp. 404-408. doi:10.1016/S0003-4975(10)63335-8

[16] I. C. Wesdorp, J. F. Bartelsman, K. Huibregtse, F. C. den Hartog Jager and G. N. Tytgat, "Treatment of Instrumental Oesophageal Perforation,” Gut, Vol. 25, No. 4, 1984 pp. 398-404. doi:10.1136/gut.25.4.398

[17] N. R. Barrett, "Report of a Case of Spontaneous Perforation of the Oesophagus Successfully Treated by Operation,” British Journal of Surgery, Vol. 35, No. 138, 1947, pp. 216-218.

[18] J. Jougon, T. Mc Bride, F. Delcambre, A. Minniti and J.F. Velly, "Primary Esophageal Repair for Boerhaave's Syndrome Whatever the Free Interval between Perforation and Treatment," European Journal Cardio-Thoracic Surgery, Vol. 25, No. 4, 2004, pp. 475-479. doi:10.1016/j.ejcts.2003.12.029

[19] R. K. Freeman, J. M. Van Woerkom, A. Vyverberg and A. J. Ascioti, "Esophageal Stent Placement for the Treatment of Spontaneous Esophageal Perforations,” The Annals of Thoracic Surgery, Vol. 88, No. 1, 2009, pp. 194-198. doi:10.1016/j.athoracsur.2009.04.004

[20] J. Saabye, H. O. Nielsen and K. Andersen, "Long-Term Observation Following Perforation and Rupture of the Esophagus,” Scandinavian Cardiovascular Journal, Vol. 22, No. 1, 1988, pp. 79-80. doi:10.3109/14017438809106056

[21] J. A. Salo, K. M. Seppälä, P. P. Pitkäranta and E. O. Kivilaakso, "Spontaneous Rupture and Functional State of the Esophagus,” Surgery, Vol. 112, No. 5, 1992, pp. 897-900.

[22] X. B. D’Journo, C. Doddoli, J. P. Avaro, P. Lienne, M. A. Giovannini, R. Giudicelli, P. A. Fuentes and P. A. Thomas, "Long-Term Observation and Functional State of the Esophagus after Primary Repair of Spontaneous Esophageal Rupture," The Annals of Thoracic Surgery, Vol. 81, No. 5, 2006, pp. 1858-1862. doi:10.1016/j.athoracsur.2005.12.050 